\title{
Beacon-Based Non-Delay Tolerant Routing Protocols for VANET: A Systematic Comparison
}

\author{
Mahmoud Ali Al Shugran ${ }^{1}$ \\ ${ }^{1}$ Department of computer seines and information technology, Jerash University, Jordan \\ Correspondence: Mahmoud Ali Al Shugran, Department of computer seines and information technology, Jerash \\ University, Jordan.
}

Received: July 28, 2021

Accepted: August 26, 2021

Online Published: August 31, 2021

doi:10.5539/mas.v15n5p12

URL: https://doi.org/10.5539/mas.v15n5p12

\begin{abstract}
A Vehicular ad hoc network (VANET) is a sort of wireless ad hoc network which are used to provide communications between nodes. The frequent topology change is considered a unique feature of VANET nature due to the high movement of its participating vehicles. Thus, the design of a routing protocol that could cope with VANET characteristics is very challenging. Position-based routing protocols are the most suitable approaches for VANET. In this paper researcher broadly discussed Beacon-based Non-Delay Tolerant Geographical-based routing protocols for VANET. The main concern is to discuss the characteristics disadvantages of those protocols. Finally, several research directions relevant to the focus of this survey are identified that define preferred features of the appropriate routing protocol that can cope with VANET challenges.
\end{abstract}

Keywords: VANET, dynamic mobility, topology changes, Beacon-based Non-Delay Tolerant

\section{Introduction}

Nodes in the VANET can communicate without the need for administration or isolated servers. Thus, VANET configuration cost is very low. VANET devoted two application categories: Safety based and non-safety based applications (Asim, R., Saira, G., Sana, A., \& Amir, Q., 2017). The safety-based vehicular applications can be subdivided into, vehicular traffic efficiency and infotainment applications (Joilson, A. J., Emilio, C., \& Wille, G., 2018). In addition to the safety applications, VANETs offered non-safety based such as real-time information to the road users like weather information, multimedia applications and mobile e-commerce. For the sake of those applications efficient and continuous exchanging of information is in need. In VANET, communication can be accomplished between vehicles directly or through multi-hop patterns. The VANET has several challenges due to its unique characteristics that may degrade the communication process between nodes (Debasis, D. \& Rajiv, M., 2018). These problems have motivated researchers to search for solutions to enhanced routing protocols. In the literature, several proposals have been issued to satisfy the new challenges and requirements of VANETs. Next, a lot of works also made a comparison between the proposed routing protocols. Those comparisons are made general evaluations. This research concentrates on the unicast geographical-based routing protocols for V2V communication for VANETs. It aims to investigate selected most popular position-based routing protocols. Thus, the research is considered unique; this is because it makes a qualitative comparison between the selected routing protocols on urban and highway environments. The rest of the paper is organized as follows; Section 2 introduces challenges and technical issues in VANETs followed by a literature review in section 3. Section 4 presents a detailed classification of unicast position-based routing protocols. Next, section 5 reviews some selected protocols belonging to the Non-Overlay position-based routing protocols. Section 6 reviews some selected protocols belonging to the Non-DTN Overlay position-based routing protocols. Section 7 concludes this research work and suggests tendencies for future research to improve the performance of routing protocols to guarantee communication reliability over VANET.

\section{Challenges and Technical Issues in VANETs}

The main goal of VANET is running vehicular ad hoc networks to provide safe and unsafe communications between the components of VANET that consider helpful to the promotion of ITS. VANET applications are widely used to meet our daily requirements. To achieve VANET goals, issues, and factors that can affect VANET should be considered. The key problems and challenges from the technical view are presented below. 
- Signal fading: Each environment that VANNET may work in has its own obstacles in addition to vehicles themselves. Existing obstacles at every environment influence the signals' strength and increasing the fading that may cause to not reach its destination node (Asim, R., Saira, G., Sana, A., \& Amir, Q., 2017).

- Bandwidth limitations. Recent years witness an increase in the types of applications in VANET. In VANETs communications between vehicles and vehicles with infrastructure are accomplished by using limited bandwidth frequency range. Also, VANET is suffering from lacking a central administrator that controls the communications between vehicles. Therefore, managing bandwidth and contention operation can't be achieved which an important impact on QoS has based routing. Hence, it is clear that there is a real need to efficiently optimize bandwidth utilization in VANET (Joilson, A. J., Emilio, C., \& Wille, G., 2018).

- Connectivity: The VANET segmentation is a regular phenomenon due to frequent link breakage between vehicles. Communication links between nodes break frequently for the reason of the high mobility feature of vehicles. To increase the QoS in VANET, it is very important to extend the life of the connection between vehicles (Debasis, D. \& Rajiv, M., 2018).

- Security and privacy. As a wireless ad hoc network, VANET can be easily penetrated by malicious observers. This is because a huge amount of information related to participating vehicles regularly transmitted. Therefore, attackers can easily make inferences about a driver's personality. Consequently, the security challenges are crucial to ensure the safety and confidentiality in VANETs. Thus, high security is very essential to guarantee the driver's and vehicle's privacy (Asim, R. Saira, G. Sana, A., \& Amir, Q., 2017).

- Routing protocol: Communication in VANET environment takes two modes; communication between vehicles (V2V) or communication between road-unit and vehicles (V2I). There are several important differences between the two environments. These variances have main influence on communication in each environment that imposes critical demand in designing an efficient routing protocol. Thus, the intended routing algorithm that will be used in VANET must adapt to the environment that will be deployed in and the communication type (Asim, R. Saira, G. Sana, A., \& Amir, Q., 2017).

\section{Literature Review}

The traditional routing protocols of wireless ad hoc networks aren't convenient to be used in VANETs because of the unique features of VANETs. Thereafter, many routing protocols have been issued to recognize VANETs' requirements. Later on, several studies were carried out to evaluate the performance of proposed routing protocols to prove they're appropriate to VANETs conditions.

Author (Singh, P., 2014, February) considered unicast and multicast routing and applied different data rates to compare their performance in VANET. The comparison is done by employing different routing metrics such as routing overhead, delay, and others to measure the performance of protocols under study. The work presented at (Sharef, B. T., Alsaqour, R. a., \& Ismail, M., 2014) considered several routing protocols with the greedy approach in the urban environment. The work came out with several issues related to routing and design strategy in VANETs. The research introduced by authors of (Manvendra, S., 2015) makes a comparison of several position-based routing protocols for VANETs. The research focused on several features of each routing protocol. The research concluded a qualitative comparison between selected protocols under study. Research executed by the authors (Ali, F., Shaikh, F. K., Ansari, A. Q., Mahoto, N. A., \& Felemban, E., 2015) studied the performance of reactive and proactive routing protocols for VANETs environments. The experiments were accomplished with the two communication modes. The study concluded that reactive protocols are more suitable to VANET compared to proactive routing protocols.

Authors (Ali, K., Phillips, I., \& Yang, H., 2016, June) provided a comparison and evaluation of three well-known routing protocols for VANET. The evaluation was achieved for the city environment setup. Three QoS metrics were used to measure the performance of compared protocols. The experiments were carried out using two different simulators. The authors concluded that position-based routing protocols outperformed topology-based protocols. The researcher (Joanne, S., 2017) presented a detailed comparison and evaluation of three topology-based and one position-based routing protocol. These protocols showed well performance with mobile ad hoc networks environments but are not suitable for VANETs environments. In the research presented by authors (Zineb, S. H. \& Imane, Z., 2017), researchers explore several routing protocols proposed in the literature, including proactive, reactive, hybrid topology-based routing protocols and position-based protocols. To evaluate the performance of selected protocols different things were considered. Thus, the study was accomplished under a various number of participating vehicles and plenty of traffics load in an urban scenario. The study concluded that position-based were more appropriate to VANETs compared to topology-based protocols. 
The authors (Amina, B. \& El Boukhari, M., 2018) presented the traditional routing protocols designed for VANETs. The study summarized the pros and cons of those protocols. Finally, researchers shed light on the open issues that need to be addressed to adapt the traditional protocol to be suitable to VANETs. Authors (Smiri, S., Boushaba, A., Ben A. R., \& Zahi, A., 2018, April) researchers compared and evaluated the performance of seven ad-hoc routing protocols for VANETs. The study considered specific routing metrics and performance metrics too. The study concluded that position-based is more convenient to VANEts compared to topology-based routing protocols. The researcher (Amina, B., Asmae, B., \& Mohamed, E., 2020) provided a detailed taxonomy of routing protocols in VANETs. The researchers explored and identified the pros and cons of each category. Next, researchers compared and evaluated selected routing protocols of each category. The evaluation is carried out by considering three quality of service metrics. The researchers concluded that position-based routing protocols may be more appropriate to VANETs by considering other routing metrics besides distance metric.

Regardless of the understanding of researchers and the different proposals to compare evaluate existing routing protocols performance in VANET. There is a shortage of in-depth performance evaluation of proposed position-based routing protocols that take into consideration the special features of VANETs. Thus, this research work compares the latest position-based that were proposed to be suited to different VANETs environments. This paper concluded a qualitative comparison between protocols under study by using comparable short summarized tables.

\section{Unicast Geographical-Based Routing Protocols in VANET}

The reliability of communication in a VANET depends on an efficient routing algorithm that can find the appropriate route between the communicating nodes (Anil, T., Kumar, T. R., \& Sivakumar, T., 2016). The formation of a route should satisfy the bandwidth consumption with minimum overhead. To accomplish communication between nodes in VANET multi-hop approach is used. There are two well-known categories for packet forwarding which are geographical-based and topology-based routing protocols (Smiri, S., Boushaba, A., Ben A. R., \& Zahi, A., 2018, April). The first category depends on two routing criteria to make routing decisions, network topology and links between communicating vehicles. Therefore, every vehicle in VANET preserves nodes routing table (NRT) to make the forwarding decision. On the other hand, the geographical-based routing category maintains a routing table that holds position information to make the forwarding decision (Harinder, K. \& Meenakshi, 2017, May). The concern of this paper focuses on available unicast position-based routing protocols.

For the geographical-based routing category, it is essential that a vehicle that has a data packet to be sent should know the current position itself as well as the destination. Thus, the routing decisions require location services such as the Global Position System (GPS) device to assign the geographic location of the participating nodes. Through using GPS, every vehicle can continuously send beacon packets with its position information. Three procedures recognize the operation of position-based routing protocols which are; Rout election, data packet sending mechanism, and the void avoidance approach.

Using a route election algorithm is optionally in geographical-based routing. Further, with location-based schemes, there are different methods to forward data packets. Greedy-forwarding is the popular approach deployed to forward data packets between two communicating nodes that are using geographical-based protocols. The recovery mode is applied as there is no appropriate next forwarding node. Based on the recovery methods geographical-based routing protocols are subdivided into three approaches; Non-Delay Tolerant Network (Non-DTN), Delay Tolerant Network (DTN), and Hybrid (Anil, T., Kumar, T. R., \& Sivakumar, T., 2016).

The forwarded packet suffers from extra delay as deploy the DTN position-based routing protocols strategy. But this strategy is suitable for delay-tolerant applications. Because of the previous reasons, the DTN is the most popular recovery approach used in designing routing protocols for VANET. Moreover, the Non-DTN protocols aim to decrease the delay in sending data packets between communicating nodes. Routing with the Non-DTN strategy has the ability to prevent carrying packets by changing routing creation. Therefore, the Non-DTN protocols are appropriate to be used with applications that can't tolerate delay. Hence, to design an appropriate Non-DTN protocol the delay routing metric should be considered. To satisfy this demand, the shortest path method is usually adopted. Nevertheless, this method sometimes introduces some delays because of holes in the network. Most current proposed Non-DTN protocols have been designed in a manner to recover this washout. The proposed solution came with two flavors; non-beacon-based, beacon-based, and hybrid protocols. The beaconless approach does not require periodically transmitting HELLO messages; Hybrid protocols integrate the beacon and beaconless methods. The concern of this paper is limited to the first category which is a beacon-based method. The beacon-based method must periodically transmit a beacon message. The time period 
to transmit the beacon varies according to the routing protocol. With this category, protocols can be classified as non-overlay and overlay (Harinder, K. \& Meenakshi, 2017, May).

\subsection{The Non-Overlay Forwarding Mechanism}

The protocols apply the Non-overlay forwarding mechanism to make forwarding decisions at each hop. The GFM is used with all protocols in the non-overlay mechanism. GFM is used to send data packets between communicating vehicles hop-by-hop. When there is no appropriate vehicle as to the next forwarded node in terms of distance, the GFM is said to fail. In case of GFM failure, the recovery mode should be applied. The RIPR (Reliability-Improving Position-Based Routing) (Ryu, M. W., 2011), Greedy Perimeter Stateless Routing with lifetime for VANETs (GPSR-L) (Sunder, A. R., Manohara P., Mounir, B., \& Joseph, M., 2008, January), and Greedy Routing with Abstract Neighbor Table (GRANT) (Sascha, S. \& Wolfgang E., 2008) are belong to this category. One of the earlier routing protocols that apply the non-overlay approach is the GPSR (Greedy Perimeter Stateless Routing) (Karp, B. \& Kung, H. T., 2000, September). Furthermore, Reliability-Based GPSR Protocol (GPSR-R) (Shelly, S. \& Babu, A. V., 2015), Greedy Perimeter Stateless Routing with Movement Awareness (GPSR-MA) (Fabrizio, G., Giulia, B., Dzmitry, K., \& Gianni, V., 2007), Greedy Perimeter Stateless Routing with Advance Greedy Routing (GPSR+AGF) (Naumov, V., Baumann, R., \& Gross, T., 2006, May), and Position-Based Routing with Distance Vector Recovery (PBR-DV) (Kirsch, B. \& Effelsberg, W., 2007) are routing protocols belong to this category.

\subsection{The Overlay Mechanism}

In the overlay mechanism, logical links that connect all nodes are constructed on the upper of other overlay network to get information such as topology of area (maps), vehicle traffic information. Hence the external information from the other networks viz. maps junctions is the point where special strategies are implemented to make forwarding decisions. Earlier routing protocols that apply the overlay approach is the GPCR (Greedy Perimeter Coordinator Routing) (Lochert, C., Mauve, M., Füßler, H., \& Hartenstein, H., 2005). Furthermore, the GpsrJ+ (Greedy Perimeter Stateless Routing Junction+) (Lee, K. C., Haerri, J., Lee, U., \& Gerla, M., 2007), November), Spatially Aware Packet Routing (SAR) (Tian, J., Han, L. \& Rothermel, K., 2003, October)., Spatial and Traffic Aware Routing (STAR) (Giudici, F. \& Pagani, E., 2005), Anchor-based Street Traffic Aware Routing (A-STAR) (Seet, BC., Liu, G., Lee, BS., Foh, CH., Wong, KJ., \& Lee, KK., 2004), Street Topology-Based Routing (STBR) (Forderer, D., 2005), Greedy Traffic-aware Routing (GyTAR) (Jerbi, M., Senouci, SM., Meraihi, R., \& Ghamri-Doudane Y., 2007, June), Enhanced Greedy Traffic Aware Routing Protocol (E-GyTAR) (Bilal, S. M., Madani, S. A., \& Khan, I. A., 2011), Enhance Greedy Traffic Aware Routing-Directional (E-GyTARD) (Bilal, S. M., Khan, A. R., \& Ali, S., 2016), The Dynamic Traffic Aware Routing (ITAR) (Huang, D., Chang, SU., Y. Yan, G., \& Xu, 2016, September), Geographic Source Routing (GSR) (Lochert, C., Mauve, M., Füßler, H., \& Hartenstein, H., 2003, July), Geographic Source Routing with Directional Forwarding Approaches (DGSR) (Bilal, S. M., Khan, A. R., \& Ali, S., 2016), Traffic flow-oriented routing (TFOR) (Abbasi, I. A. Nazir, B., \& Abbasi, A. 2014), Landmark Overlays for Urban Vehicular Routing Environments (LOUVRE) (Lee, K. C., Le, M., Harri, J., \& Gerla, M., 2008, September), Directional Greedy Routing (DGR) (Gong, J., Xu, C., \& Holle, J., 2007, June), Predictive Directional Greedy Routing (PDGR) (Gong, J., Xu, C., \& Holle, J., 2007, June), Directional Greedy routing protocol (DGRP) (Kumar, R. \& Rao, S. V., 2008, December), Reliable Directional Greedy Routing (RDGR) (Prasanth, K., Duraiswamy, K., Jayasudha, K., \& Chandrasekar, C., 2010), The Connectivity-Aware Routing (CAR) (Naumov, V., \& Gross T. R., 2007, May), Position Based Density Conscious Routing (PBDCR) (Ram, A. \& Mishra, M. K., 2017) are routing protocols belong to this category.

\section{Non-Overlay Position-Based Routing Protocols}

As shown in Figure 1, this part presents several unicast geographical-based routing protocols and more precisely the Non-DTN Non-Overlay for both environments urban and highway. Further, Table 1 presents a comparison of the surveyed non-overlay geographical protocols for the two environments. 


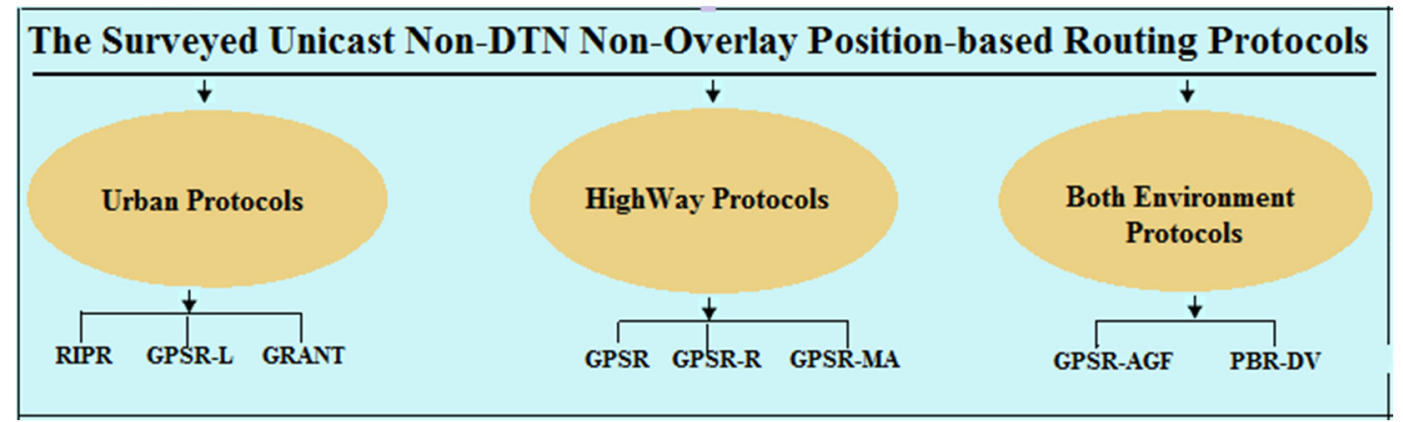

Figure 1. The surveyed non-DTN non-overlay protocols

\subsection{Representative Protocols in Urban Environment}

\subsubsection{A Reliability-Improving Position-Based Routing (RIPR)}

The RIPR is presented to solve the problem of a void problem and link failure. The proposed protocol is the RIPR (reliability-improving position-based routing). The RIPR algorithm consists of a greedy mode and avoidance of voids approach. The RIPR algorithm finds out the neighbor positions based on velocities and moving directions of vehicles. Then, the forwarding decision is made up for sending packets to the final target. Thus, the RIPR algorithm can minimize the chances of being stuck in a void problem.

\subsubsection{Greedy Perimeter Stateless Routing with Lifetime (GPSR-L)}

In GPSR implementation, there was the main issue, a given node selects a neighbor that is no longer exists when in reality it remains present in its coverage area. To deal with such issues, Greedy Perimeter Stateless Routing with Lifetime (GPSR-L) is introduced. With GPSR-L, the concept of link quality and the lifetime are adopted. Hence, the selection decision for the next forwarder vehicle can be more reliable. This derives a suitable choice of the next-forwarder vehicle, thus minimizing packet loss. The experiment outcomes demonstrated that GPSR-L performs better than GPSR in several performance metrics such as packet delivery ratio (PDR).

\subsubsection{Greedy Routing with Abstract Neighbor Table (GRANT)}

To prevent the void problems in GPSR to occur, a new routing concept is proposed. The new proposal is called Greedy Routing with Abstract Neighbor Table (GRANT). With GRANT, each vehicle in the network maintains a routing table called Abstract Neighbor Table (ANT). The table holds information about the surrounding neighbors existing at $\mathrm{x}$-hops. The ANT divides the network into areas. Each area includes a commissary neighbor for only one area. Thus, overhead incurred by beacon messages could be reduced. Every vehicle knows the best route to be selected. Every vehicle applying the GRANT algorithm broadcasts its ANT in the beacon message. As a vehicle receives a hello message, it calculates the area belong to the vehicle that makes the transmitting and its neighbors. Next, it classifies them into several hops from the present vehicle.

\subsection{Representative Protocols in Highway Environment}

\subsubsection{Greedy Perimeter Stateless Routing (GPSR)}

GPSR is a simple Non-DTN geographical-based protocol for VANETs. It represents the cornerstone of several protocols in VANET. The GPSR algorithm allocates the source vehicle using a global position system. The geographical location of other participating nodes is determined by exploiting beacon messages. Furthermore, the GPSR uses location services to determine the geographical location of the final target node. The functionality of GPSR depends on two modes: the GFM mode, and the recovery mode. In GFM, the next forwarder node is chosen by considering the distance routing metric. The packet is forwarded hop-by-hop until it reaches its final target. In case the forwarded packet suffers from a void problem, then the GPSR algorithm tune to the second mode. The GPSR switches to the second operation by using perimeter mode by applying the right-hand rule to route around voids.

\subsubsection{Reliability-Based GPSR Protocol (GPSR-R)}

A new approach to estimate link lifetime and hired to design the called GPSR-R (Reliability-based GPSR). With the GPSR-R protocol, the vehicle sends beacon messages (BMs) to its neighbors periodically. The beacon has extra data to be used in making forwarding decisions that are the current velocity characteristics (vehicle speed and motion direction) of the node that transmits the BM. The node which receives the beacon builds its Neighbor Routing Table (NRT) based on the content of the BMs. The GPSR-R algorithm makes a selection decision of the next forwarded vehicle based on the weight of the link lifetime. If there is no candidate next relay node, the 
vehicle switches to the perimeter mode. Authors have approved that GPSR-R outperforms GPSR and GPSR-L protocols in terms of packet delivery ratio and throughput.

\subsubsection{Greedy Perimeter Stateless Routing with Movement Awareness (GPSR-MA)}

An extension for the well-known GPSR protocol called Greedy Perimeter Stateless Routing with Movement Awareness (GPSR-MA). GPSR-MA employs a set of routing metrics for making a forwarding decision. Those routing metrics are the velocity characteristics (vehicle speed and motion direction). The GPSR-MA processing is the same as the original GPSR features. But, as the proposed algorithm deployed several criteria to select the next relay node, thus constructed route considers the most stable route from source to destination. GPSR-MA algorithm can be a good choice for the dynamic scenario. GPSR-MA outperformed the original GPSR in terms of several performance metrics.

\subsection{Representatives Protocols in Urban and Highway Environments}

\subsubsection{GPSR+AGF (Advanced Greedy Forwarding)}

The AGF routing protocol is proposed to overcome the two main problems with GPSR. The first problem case is represented by the stale input data around the location of the surrounding vehicle in Neighbor Routing Tale (NRT). The second problem case is represented by having untrue data around the location of the final target in the header of the packet. To solve those issues, in AGF, nodes periodically broadcast Hello Messages, in addition to its current location, the message includes the velocity characteristics (vehicle speed and motion direction), and the total time that the packet spent between sender and recipient. Further, it contains the packet processing time, up to the actual sending vehicle within the stream. This data is used to build the NRT that is used to find out the next forwarding node.

\subsubsection{Position-Based Routing with Distance Vector Recovery (PBR-DV)}

The PBR-DV algorithm employed the advantage of two main routing approaches which are the greedy position-based routing strategy and the reactive topology-based routing protocol recovery approach. With PBR-DV participating vehicles occasionally transmit a hello message. The transmitted packet holds information about the vehicle's geographical location and its identity. The superior idea of PBR-DV starts when a packet falls into the void. At this point, the PBR-DV protocol switches to recovery mode of the reactive topology-based protocol to forward the data packet.

Table 1. Non-Delay Non-Overlay position-based routing protocols

\begin{tabular}{|c|c|c|c|c|c|c|c|c|c|}
\hline Protocol & GPS & L.S & $\begin{array}{l}\text { Neighborhood } \\
\text { Beacon }\end{array}$ & F.S. & R.S. & $\mathbf{V} 2 \mathrm{~V}$ & T.A & M.B. & O.A \\
\hline \multicolumn{10}{|c|}{ Highway Environment } \\
\hline GPSR & $\checkmark$ & $\checkmark$ & $\checkmark$ & greedy & Right-hand rule & $\checkmark$ & $x$ & $\checkmark$ & $x$ \\
\hline GPSR-R & $\checkmark$ & $\checkmark$ & $\checkmark$ & greedy & Right-hand rule & $\checkmark$ & $x$ & $\checkmark$ & $x$ \\
\hline GPSR-MA & $\checkmark$ & $\checkmark$ & $\checkmark$ & greedy & Right-hand rule & $\checkmark$ & $x$ & $\checkmark$ & $x$ \\
\hline \multicolumn{10}{|c|}{ Urban Environment } \\
\hline RIPR & $\checkmark$ & $\checkmark$ & $\checkmark$ & greedy & Right-hand rule & $\checkmark$ & $\checkmark$ & $\checkmark$ & $\checkmark$ \\
\hline GPSR-L & $\checkmark$ & $\checkmark$ & $\checkmark$ & greedy & Right-hand rule & $\checkmark$ & $x$ & $\checkmark$ & $x$ \\
\hline GRANT & $\checkmark$ & $\checkmark$ & $\checkmark+\mathrm{x}$-hops & $\begin{array}{l}\text { Based on } \\
\text { metrics }\end{array}$ & $\begin{array}{l}\text { Face-2, distance } \\
\text { vector-based }\end{array}$ & $\checkmark$ & $x$ & $\checkmark$ & $\checkmark$ \\
\hline
\end{tabular}

\section{Urban and highway}

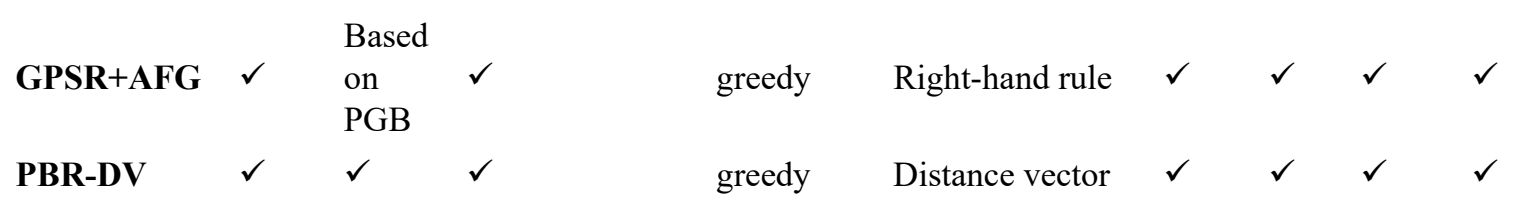

L.S.: Location Service, F.S.: Forwarding Strategy, R.S.: Recovery strategy, M.M.: Mobility Model, T.A.: Traffic Awareness, M.B.: Map-Based, O.A.: Obstacles Awareness 


\section{Non-DTN Overlay Location-Based Category}

This section distinguishes the unicast Non-DTN Overlay location-based approaches for both environments (urban and highway). Figure 2 presents the surveyed protocols in both environments. Further, Table 2 presents a comparison of the surveyed protocols relay on the two environments.

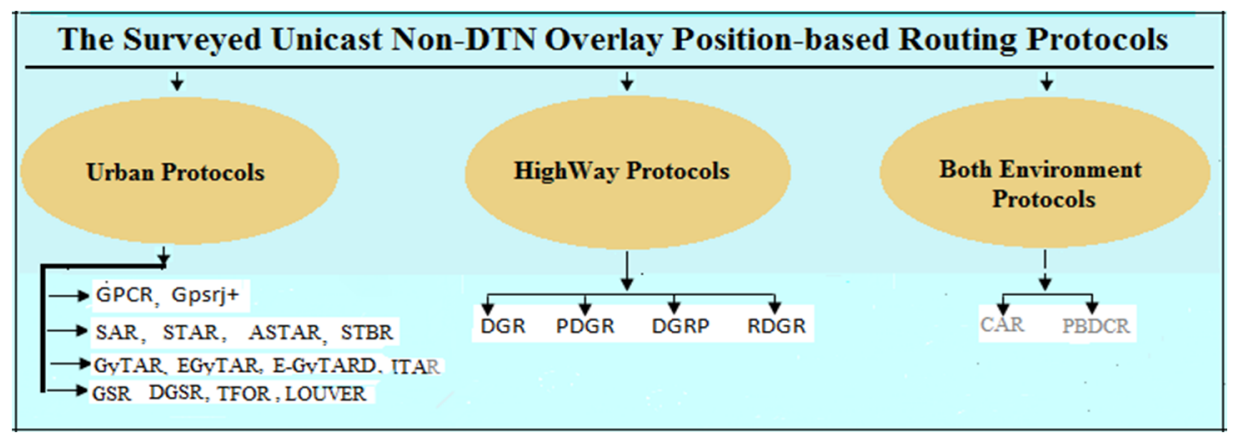

Figure 2. The surveyed Non-DTN overlay location-based approaches

\subsection{Representatives Protocols in Urban Environment}

\subsubsection{Greedy Perimeter Coordinator Routing (GPCR)}

Greedy perimeter coordinator routing (GPCR) is a V2V protocol used in an urban environment. It is a street-aware protocol. GPCR algorithm performs two strategies to find the optimal routes. The strategies are the restricted greedy forwarding (RGF) on the basis of the prior selected route, and the perimeter mode. GPCR is like other geographical-based protocols, it has GPS and the ability to know destination location using a location service program. GPCR algorithm takes its decision to select the next junction instead vehicle. With the GPCR algorithm, the vehicle which holds the data packet sends it to the next junction using the restricted forwarding approach. In GPCR, when the RGF fails to forward the data packet i.e. packet fall in the void, it applies recovery mode to overcome such problem. The recovery mode applies the right-hand rule to assign which junction the packet should be forwarded to. However, GPCR suffers from several issues that need to solve. Firstly, the delay time increase as using restricted greed based on density criterion. Secondly, the RGF mode requires more hops to reach the final destination. Thirdly, as restricted greed often failed, recovery mode will be used more often too that incurs extra delay.

\subsubsection{Greedy Perimeter Stateless Routing Junction+ (GpsrJ+)}

GpsrJ+ is a V2V protocol proposed to be used in an urban environment. GpsrJ+ is designed to improve GPCR performance based on the minimal modification. GpsrJ+ algorithm constructed of two methods; special greedy forwarding method (GFM) and GpsrJ+'s recovery method. The packet holder uses GFM to send the packet along the route segment. Vehicles get the road segment extracted from beacon messages received from neighbors. GpsrJ+ uses digital maps to select the next forwarded node which results in reduces the void problem. In such a case the algorithm of the protocol has two choices; the first case is applied as both the road segment and the pre-computed segment are identical thus packet is sent to the furthest neighbor. The second case will be applied as both the road segment and the pre-computed segment are different, thus the packet is sent to the junction neighbor. In case the coordinator vehicle moves in the opposite direction from the actual forwarding vehicle, it chooses the coordinator vehicle as a suitable pass on it. Appropriately, the main forwarding rosolutions are made at junctions. As the data packet can't find the next forwarder closer to the destination, the algorithm switches to the recovery mode.

\subsubsection{Spatially Aware Packet Routing (SAR)}

The SAR algorithm implements spatial awareness for packet forwarding. With the SAR, a vehicle gets its position on the circumstantial model. SAR algorithm calculates the shortest path between two communicating vehicles by using a static street map that is extracted by using Geographic Information Systems (GIS). In case the algorithm fails to discover a suitable vehicle along the forwarding route; the SAR algorithm will employ one of two forwarding strategies. Those strategies are postponement that is activated to settle down the data packet, the other strategy is to send the data to its final target by using the greedy approach. Both strategies suffer from several drawbacks; the suspension buffer approach incurs misleading information in the vehicle's neighbors' routing table and high delay. Also using the second strategy might cause to suffer from the void problem. Furthermore, both alternative strategies don't take into account another routing criterion such as vehicular traffic density. 


\subsubsection{Spatial and Traffic Aware Routing (STAR)}

With the Spatial and Traffic-Aware Routing (STAR) protocol algorithm, vehicles construct the traffic table based on hello messages. The vehicle periodically broadcasts a beacon packet. This beacon holds information from the sender routing table or neighbors' traffic table such as; the sender identity, its location, as well as its vehicular traffic status. With the STAR algorithm, the node finds paths on the need-base by using its routing table. A vehicle finds the closest way to the final target by applying the Dijkstra algorithm. Moreover, the STAR algorithm selects streets with queues of vehicles because they can come up with many other options for data forwarding, thus shrinking the chances of the void problem. With STAR, a forwarding path may be tuned dynamically to find exact traffic information. However, in STAR using the beaconing approach may cause bandwidth problems.

\subsubsection{Anchor-Based Street Traffic Aware Routing (A-STAR)}

The Anchor-based Street Traffic-Aware Routing (A-STAR) uses street maps that assist in finding the series of crossroads through which the data forwarded to the destination. A-STAR algorithm enhanced GSR functionality by considering the means of vehicle traffic located in route. With the A-STAR algorithm, the vehicle which has a data packet to be sent finds the forwarding route by using the Dijkstra algorithm then it forwards the data packet by using a greedy forwarding approach along the route. The greedy approach is used to send packet between two junctions. In case of a void problem occurrence, A-STAR uses an efficient local recovery strategy to solve the void problem. However, beacon messages don't hold enough information about vehicles. A-STAR algorithm doesn't take into account other criteria when finding out the optimal path to select the appropriate next relay node. These criterions such as neighbors' speed and motion direction are very essential in making routing decisions. The shorten knowledge about neighbors may result in a bad decision to select the next forwarded node that may degrade the performance of the routing protocol.

\subsubsection{Street Topology-Based Routing (STBR)}

The STBR uses a street map and location information address to forward packets between communicating vehicles. The vehicles use a location service to know the location of the final targeted vehicle. With STBR, nodes construct their own routing table based on beacons messages. The STBR algorithm marks nodes at a junction as the master node, slave node, and forwarder node. Every master maintains two junction neighbor tables. If a vehicle needs to forward a data packet, it evaluates the optimal shortest route to the final targeted node. All junctions on this optimal route are included in the header of the data packet. A vehicle that receives the data packet has the ability to choose the neighbor that satisfies the forwarding condition. The selected one must be the closest to the destination to be the next selected node. After the packet arrives at the junction, the junction is deleted from the packet header and the position of the next one is used as a new targeted node. After the last junction is reached, the location of the final target vehicle is selected. The main drawbacks of STBR are; Firstly, the protocol is not convenient for combined scenarios because it would try to send hello packets to the junction along a highway. Secondly, the complexity is growing when applying STBR algorithm especially when the protocol is deployed in private conditions like transferring the two-hop neighbor table to the new master when the old master goes away from the junction.

\subsubsection{Greedy Traffic-Aware Routing (GyTAR)}

The GyTAR is proposed for the urban areas it is a junction-based routing protocol that employs a digital map. GyTAR algorithm exploits the digital map to construct robust routes. GyTAR successes to decrease the total bandwidth by minimizing control messages to the lower rate. Further, it aims to minimize data packet loss and delay. GyTAR as other position-based routing protocols participating nodes should know their position also the destination location. GyTAR has two modes: the first mode is finding out the suitable shortest route by applying a dynamic crossroads choosing technique through using traffic density metric and curve-metric distance to the destination. The second mode is an enhanced greedy forwarding that sends the packet from one communicated crossroads to another. With the first mode; the algorithm selects the connection by applying the two routing metrics; traffic density and curve-metric distance to the final targeted node. The weight value of the routing metrics is put to all neighboring junctions to the targeted location. The junction that satisfies the condition of the two metrics is selected to be the next hop. If the rotted packet reaches the void, then the algorithm directly switches to the carry-and-forward technique. The main issues in GyTAR are: firstly GyTAR approach does not consider the direction of the vehicle while selecting the next junction that results in some extra delay. Secondly, GyTAR suffers from a local optimum problem that also results in extra delay. AS the total outcome of those issues, the performance of the network may be degrading. 


\subsubsection{Enhanced Greedy Traffic Aware Routing Protocol (E-GyTAR)}

E-GyTAR is an improvement protocol to GyTAR. Participating nodes that apply E-GyTAR, must know their location also destination position to make a forwarding decision. Further, participating nodes should periodically transmit Hello Message (HM). Each participating node keeps a Neighbour Routing Table (NRT). The NRT holds information about neighbors, such as the neighbor's current position, velocity. Further, NRT is regularly renewed by using the datum received in HM. As the node needs to communicate with a targeted destination then it refers to its NRT to discover the optimal route. E-GyTAR algorithm deploys two stages as GyTAR algorithm. The E-GyTAR algorithm depends on the number of nodes that make moves towards the destination to select the optimal path. In E-GyTAR, if the packet reaches the void, then the algorithm switches to the recovery mode that is the carry and forward technique. However, the selection method to find a suitable bath considers the main drawback of the E-GyTAR routing protocol. Furthermore, E-GyTAR covers to carry and forward technique which results in more delays that degrades the performance of the network.

\subsubsection{Enhance Greedy Traffic Aware Routing-Directional (E-GyTARD)}

E-GyTARD is an enhanced novel of E-GyTAR with Directional Greedy Forwarding (DGF). E-GyTARD algorithm can discover the geographical location of destination by the means of using location services. E-GyTARD deploys the DGF approach and Crossroad Chosen approach. Nodes deploy the E-GyTARD algorithm forward the packets between junctions using DGF strategy and shortest distance to the final target. In E-GyTARD, if the forwarded data packet reaches the void, then the E-GyTARD algorithm switches to recovery mode that is the carry and forward technique. The main issue associated with the E-GyTARD routing protocol is that converts to carry and forward technique results in more delay that degrade the performance of the network.

\subsubsection{Dynamic Traffic Aware Routing (ITAR)}

ITAR is an improved version of E-GyTAR. ITAR algorithm improves junction selecting strategy and recovery strategy used by E-GyTAR. With ITAR, each vehicle has GPS and location service which is Grid Location Service (GLS). Further, each participating vehicle has digital maps and Vehicular traffic estimation techniques like IFTIS on board. ITAR selects the junctions that will construct the routing path dynamically. As a source vehicle has a data packet that needs to be sent, and a packet is at a junction, the neighboring junction was targeted. The next relay junction is selected based on the score value. To make the forwarding decision, the algorithm of ITAR optimizes the weight of the two routing metrics. Packets are forwarded by applying an improved greedy forwarding manner as E-GyTAR does. When a forwarded data packet encounters a void problem, the ITAR algorithm applies a conditional carry and forward approach.

\subsubsection{Geographic Source Routing (GSR)}

The GSR algorithm combines that are geographical-based routing and topological knowledge. The reactive location service (RLS) is deployed by the protocol to obtain information concerning the final destination's geographical location. Participating nodes that have data to be sent, use a city map after it is converted to city topology view. With the GSR algorithm data packet is forwarded anchor (junction) by anchor (junction) to reach the final target node. Those anchors shape the optimal route in terms of distance. To forward data packets from one to another anchor, the greedy forwarding approach is deployed. When the data packet is reach void, the GSR algorithm switches to a carry and forward strategy. It can be noticed that the GSR algorithm didn't use vehicular traffic information when finding out the optimal route and this is the main issue of the GSR algorithm. Also, the source vehicle that needs to send a data packet selects a fixed intersection which shapes the optimal route that incurred bad performance in extremely mobile VANET.

\subsubsection{Geographic Source Routing with Directional Forwarding Approaches (DGSR)}

The DGSR is an improved novel of GSR routing protocol. In DGSR, the vehicles should get the location of the final target vehicle via LS (location service). The DGSR protocol algorithm employs the Dijkstra algorithm to find out the shortest path to the final target vehicle. The optimal route is constructed of a series of road intersections based on the distance metrics. Any data packet forwarded from the sender vehicle should follow the optimal path to reach its final destination. The new improvement to the DGSR algorithm is that the forwarding node uses DGF to send data packets to the final targeted vehicle. In case the forwarded packet is stuck in the void, the DGSR algorithm switches to recovery mode that is the carry and forward strategy. However, the DGSR protocol does not take into account the condition of the link while routing the data packet. Therefore, as vehicle speed increase the opportunity of link breakage increases too due to a high topology change. 


\subsubsection{The Traffic Flow-Oriented Routing (TFOR)}

The TFOR participating vehicles need to know their geographic location in addition to the geographical location of the final targeted vehicle. Thus, all nodes need to employ the global positioning system and location service. The TFOR algorithm is composed of two approaches to accomplish its function. The first mechanism is used to select optimal crossroads base on specific criteria. The second mechanism depends on using two-hop neighbor information to construct its routing table to accurately forward data packets. Further, the vehicle that has a data packet to be sent should find out neighbors' junctions by employing a digital city map. Thereafter the road intersection that has the shortest distance to the final targeted vehicle will be selected as the next hop. In TFOR the real-time directional and non-directional traffic density is gained to discover the most optimal route. If the packet reaches void i.e. packet holder does not have a vehicle towards the final targeted vehicle then it applies to carry and forward recovery mode.

\subsubsection{Landmark Overlays for Urban Vehicular Routing Environments (LOUVRE)}

The LOUVRE optimal route is constructed of the joint roads in the VANET. Thus, a forwarding route from a sender node to the final receiver node is determined by the means of the overlay. LOUVRE consider as a point-to-point strategy to find out and provide traffic density information of every route. In LOUVER, vehicles keep a link-state table that contains information for routing between overlay nodes represented by junctions. Further, Dijkstra's routing search algorithm is used to build the overlay link-state routing table. Sours node that has a data packet to be forwarded estimates the traffic load to straighten the presence of an overlay link participating vehicles that apply LOUVRE employ an onboard navigator system to provide a map of the area. This map is employed to build a road topology graph (RTG). The RTG consist of existing roads as vertices and edges between those vertices that show that they are connected together. Compared to other routing protocols, due to overlay links LOUVRE is considered obstacle-free. Further, LOUVRE algorithm minimizes the opportunity of falling into a void problem.

\subsection{Highway Environment}

\subsubsection{Directional Greedy Routing (DGR)}

With Directional Greedy Routing (DGR), Participating vehicles use the DGR algorithm hires static maps and LS (location services) to obtain the location information of the final targeted vehicle. In addition, all vehicles must know how to get to their current location as well as their speed and motion direction. The DGR algorithm employs DGF (Directional Greedy Forwarding) for sending the packets towards the destination. In case, a sender vehicle needs to forward the data packet to a final target vehicle, the criterion to choose the next relay node is based on distance and motion direction routing metrics. DGR algorithm chooses a vehicle that is closest to the target node and is moving towards the target. Furthermore, if the packet holder does not have the vehicle in the direction of the destination then it switches to carry and forward recovery mode. Using recovery mode is for certain specified time duration as threshold and tries to find out the next vehicle moving in the direction of the destination. The DGR Routing scheme mediates to minimize the routing loops problem. However, based on DGR functionality it may incur more delay compared to other protocols.

\subsubsection{Predictive Directional Greedy Routing (PDGR)}

The PDGR is an enhanced version of DGR. The PDGR algorithm is able to forward packets to the most suitable next hop based on both current and predictable future situations. With the PDGR algorithm, participating nodes use static digital maps and GPS services to find out their precise position. PDGR applies Direction First Forwarding (DFF) strategy and Position First Forwarding (PFF) strategy. Hence, the weighted score is computed from these two strategies. The weighted score is estimated for the source node and its current neighbors, and potential neighbors. When selecting the next appropriate hop, the PDGR algorithm considers both position and direction in making its decision. The next hop is selected by calculating the weighted score of the two metrics. Additionally, a prediction approach is applied by considering the packet carrier's possible future neighbors to make routing more efficient in PDGR. If the forwarded data packet reaches void i.e. packet holder does not have a vehicle in the direction of the destination then it converts to carry and forward recovery mode until it finds out the next vehicle moving towards a final target destination. The main drawback of the PDGR is the network disconnection problem if the predicted node is selected as the next relay node that may become out of sender range.

\subsubsection{Directional Greedy routing protocol (DGRP)}

The DGRP uses the two forwarding strategies which are; greedy and perimeter. Further, the DGRP algorithm depends on the DGR protocol with more improvements. The enhancement to the DGRP algorithm is 
accomplished by predicting the position of the vehicle's neighboring nodes during the beacon interval. To predict the new positions, the DGRP algorithm uses the neighbors' two routing metrics that are speed and direction information provided in beacon packets. A source node that has a data packet to be forwarded predicts the position of its neighbor's vehicles within the beacon interval. Next, it selects the most suitable next forwarder vehicle. The selection process is depending on choosing the vehicles which are near the final target or to the next intermediate vehicle. The main drawback of DGRP: that each participating vehicle has to find out the routing metrics to make a prediction process that incurs more delay in VANET.

\subsubsection{Reliable Directional Greedy Routing (RDGR)}

The RDGR algorithm depends on the DGR protocol with more improvements to increase its reliability. Vehicles that apply the RDGR approach obtain the position of their neighboring nodes from GPS. Further, neighbors' movement information is excluded from received beacon messages. The RDGR algorithm uses information of vehicles' position, speed, the direction of motion i.e. movement information to predict the link stability (LS) of their neighbors. The RDGR algorithm finds out neighbors LS in a dispense fashion for reliable forwarding of the data packets. The packet sender uses the neighbor's LS to choose the most appropriate next forwarder vehicle. The RDGR algorithm applies four routing metrics that are; distance to destination, movement information, and neighbor's LS. Those routing metrics are used to find out the most suitable next forwarder vehicle. The RDGR algorithm combines a possibility score-based design, which reduces the breaks of communicating liks, and improves the packet delivery ratio and reliability of the route.

\subsection{Urban and Highway Environments}

\subsubsection{The Connectivity-Aware Routing (CAR)}

With CAR every node in the network sends periodic beacon messages that contain its movement information (velocity). Vehicles construct their neighborhood table (NHT) based on received beacon messages. An entry in the NHT expires and is removed when the distance between nodes exceeds a sill amount. The CAR algorithm constructed of four main components. In the first two parts; the algorithm combines finding the position of the final targeted vehicle with detecting the connected routes between communicating vehicles. The route that comes up with the best reliability and the minimum delays is selected based on employed routing metrics. In the second two parts; the CAR algorithm starts the record of keeper which is a geographic sign letter, it is saved and forwarded node by node to transmit the packet content. The CAR algorithm is also composed of two void avoidance approaches to overcome the void problem. The main drawback of the CAR algorithm is that the data packet should know the whole path until it reaches the final destination. This process is not suitable for VANET due to rapid topology changes. Further, the broadcasting that is used in routing discovery increases extra overheads and incurs network congestions.

\subsubsection{Position Based Density Conscious Routing (PBDCR)}

With PBDCR, the source vehicle employes location service to discover the destination's geographic position Further, each vehicle in PBDCR gan get its position by the means of using Global positioning systems (GPS). In addition, vehicles can get the street-level information through the use of the pre-loaded digital map by using an onboard navigation system. As a source vehicle has a packet to be forwarded it first obtains the geographical location of the destination by using LS (location service). Next, it selects the optimum forwarder node by using the PBDCR algorithm. If it fails to find the next vehicle as optimum forwarder based on the algorithm selection criteria. Then a source vehicle handover the packet to the vehicle in its communication range toward to the destination which has less absolute velocity difference. Furthermore, if the packet holder does not have the vehicle in the direction of the destination then it carries the packet for certain specified time duration as threshold and try to find out the next vehicle in its communication range. The main drawbacks of PBDCR; the end-to-enddelay may increases due to selection process and extra delay is gained with the recovery mode. Moreover, vehicles move in opposite direction with forwarded vehicle didn't consider in the selection process. 
Table 2. Non-Delay Overlay position-based routing protocols

\begin{tabular}{|c|c|c|c|c|c|c|c|c|c|}
\hline 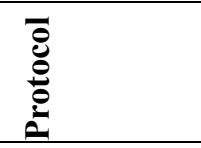 & ڤ̂. & $\dot{\dot{\leftrightarrow}}$ & 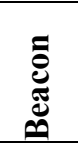 & $\dot{\dot{Q}}$ & $\dot{\dot{\theta}}$ & 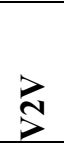 & 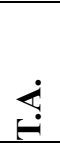 & $\stackrel{\infty}{\dot{\Sigma}}$ & i \\
\hline \multicolumn{10}{|c|}{ Urban Environment } \\
\hline GPCR & $\checkmark$ & $\checkmark$ & $\checkmark$ & Restricted greedy & Right-hand rule & $\checkmark$ & $x$ & $\checkmark$ & $\checkmark$ \\
\hline Gpsrj+ & $\checkmark$ & $\checkmark$ & $\checkmark$ & $\begin{array}{l}\text { Enhanced } \\
\text { Restricted greedy } \\
\text { (prediction-based) }\end{array}$ & Right-hand rule & $\checkmark$ & $x$ & $x$ & $x$ \\
\hline GyTAR & $\checkmark$ & $\checkmark$ & $\checkmark$ & $\begin{array}{l}\text { Advanced greedy, } \\
\text { intersection-based }\end{array}$ & Carry and forward & $\checkmark$ & $\checkmark$ & $\checkmark$ & $\checkmark$ \\
\hline EGyTAR & $\checkmark$ & $\checkmark$ & $\checkmark$ & $\begin{array}{l}\text { Improvedgreedy } \\
\text { forwardin }\end{array}$ & Carry and forward & $\checkmark$ & $\checkmark$ & $\checkmark$ & $\checkmark$ \\
\hline EGyTAR-D & $\checkmark$ & $\checkmark$ & $\checkmark$ & Advanced greedy & Carry and forward & $\checkmark$ & $\checkmark$ & $\checkmark$ & $\checkmark$ \\
\hline ITAR & $\checkmark$ & $\checkmark$ & $\checkmark$ & Advanced greedy & $\begin{array}{l}\text { Conditional Carry } \\
\text { and forward }\end{array}$ & $\checkmark$ & $\checkmark$ & $\checkmark$ & $\checkmark$ \\
\hline GSR & $\checkmark$ & $\checkmark$ & $\checkmark$ & $\begin{array}{l}\text { Greedy along the } \\
\text { shortest path, } \\
\text { intersection-based }\end{array}$ & $\begin{array}{l}\text { Switches back to } \\
\text { greedy mode }\end{array}$ & $\checkmark$ & $x$ & $\checkmark$ & $\checkmark$ \\
\hline DGSR & $\checkmark$ & $\checkmark$ & $\checkmark$ & $\begin{array}{l}\text { Directional } \\
\text { Greedy } \\
\text { Forwarding }\end{array}$ & Carry and forward & $\checkmark$ & $x$ & $\checkmark$ & $\checkmark$ \\
\hline TFOR & $\checkmark$ & $\checkmark$ & $\checkmark$ & $\begin{array}{l}\text { Improved greedy } \\
\text { forwarding based } \\
\text { on } 2 \text { hops }\end{array}$ & Carry and forward & $\checkmark$ & $\checkmark$ & $\checkmark$ & $\checkmark$ \\
\hline LOUVER & $\checkmark$ & $\checkmark$ & $\checkmark$ & - & - & $\checkmark$ & $\checkmark$ & $\checkmark$ & $\checkmark$ \\
\hline SAR & $\checkmark$ & $\checkmark$ & $\checkmark$ & Spatial aware & $\begin{array}{l}\text { Suspends and buffer } \\
\text { the packet }\end{array}$ & $\checkmark$ & $\checkmark$ & $\checkmark$ & $x$ \\
\hline STAR & $\checkmark$ & $\checkmark$ & $\checkmark$ & $\begin{array}{l}\text { Greedy } \\
\text { forwarding }\end{array}$ & Compute new route & $\checkmark$ & $x$ & $\checkmark$ & $\checkmark$ \\
\hline A-STAR & $\checkmark$ & $\checkmark$ & $\checkmark$ & $\begin{array}{l}\text { Greedy along the } \\
\text { anchor path }\end{array}$ & $\begin{array}{l}\text { Compute a new } \\
\text { anchor path }\end{array}$ & $\checkmark$ & $x$ & $\checkmark$ & $\checkmark$ \\
\hline STBR & $\checkmark$ & $\checkmark$ & $\mathrm{J} 2 \mathrm{~J}$ & - & - & $\checkmark$ & $\checkmark$ & $\checkmark$ & $\checkmark$ \\
\hline \multicolumn{10}{|c|}{ Highway Environment } \\
\hline DGR & $\checkmark$ & $\checkmark$ & $\checkmark$ & $\begin{array}{l}\text { Greedy } \\
\text { Forwarding }\end{array}$ & Carry and forward & $\checkmark$ & $\checkmark$ & $\checkmark$ & $x$ \\
\hline PDGR & $\checkmark$ & $\checkmark$ & $\checkmark$ & $\begin{array}{l}\text { Greedy } \\
\text { Forwarding }\end{array}$ & Carry and forward & $\checkmark$ & $\checkmark$ & $\checkmark$ & $\checkmark$ \\
\hline DGRP & $\checkmark$ & $\checkmark$ & $\checkmark$ & $\begin{array}{l}\text { Greedy } \\
\text { Forwarding }\end{array}$ & Carry and forward & $\checkmark$ & $x$ & $\checkmark$ & $x$ \\
\hline RDGR & $\checkmark$ & $\checkmark$ & $\checkmark$ & $\begin{array}{l}\text { Greedy } \\
\text { Forwarding }\end{array}$ & Carry and forward & $\checkmark$ & $x$ & $\checkmark$ & $x$ \\
\hline \multicolumn{10}{|c|}{ Urban and Highway Environments } \\
\hline CAR & $\checkmark$ & $\checkmark$ & $\checkmark$ & $\begin{array}{l}\text { Greedy along the } \\
\text { shortest path, } \\
\text { intersection-based }\end{array}$ & $\begin{array}{l}\text { Switches back to } \\
\text { greedy mode }\end{array}$ & $\checkmark$ & $\checkmark$ & $\checkmark$ & $\checkmark$ \\
\hline PBDCR & $\checkmark$ & $\checkmark$ & $\checkmark$ & $\begin{array}{l}\text { Restricted greedy } \\
\text { forwarding }\end{array}$ & carry the packet & $\checkmark$ & $\checkmark$ & $\checkmark$ & $\checkmark$ \\
\hline $\begin{array}{l}\text { L.S.: Locatio } \\
\text { Traffic Awar } \\
\text { junction to ju }\end{array}$ & & & 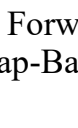 & $\begin{array}{l}\text { ding Strategy, R.S.: } \\
\text { d, O.A.: Obstacles A }\end{array}$ & $\begin{array}{l}\text { Recovery strategy, M.N } \\
\text { lwareness, NeiH-B: nei }\end{array}$ & & & & \\
\hline
\end{tabular}




\section{Conclusion and Future Perspective}

In this paper, researcher has reviewed existing beacon-based unicast routing protocols. Aforementioned table showed a detailed comparison of surveyed protocols. Researcher presented the mechanism of each protocol and described the advantages and disadvantages of each one. Moreover, a detailed performance comparison of these protocols has been conducting. It was noticed that the deployed forwarding strategy is usually in greedy or optimum approach. Greedy forwarding depends on the local information to make the routing decision. On the other hand, optimum forwarding strategies account on the entire information in a network to select the best routing path. Recovery strategy is about route recovery approach in case of the failure of selected routing path. Based on our study researcher conclude that the designing of Unicast position-based routing protocols still have many issues to be solved. Thus, the designing an efficient routing protocols for VANET is one of the major challenges to be attained. Therefore, future research for designing efficient routing protocols for VANET should account the following requirements: Compatibility: To design an intelligent and optimized position-based routing protocol that can be adapt with several environments. For example, to design a routing protocol in an urban, the interference should be considered. Scalability: To design an efficient routing protocol that should scale well with the increase in the number of users on road. Also, the intended protocol should be designed with min-delay under low network density. Efficiency: To design an efficient routing protocol that should adapt to different traffic scenarios seamlessly. Also, it should take into account the effect of extra use of network' resources. Robustness: To design an efficient routing protocol that should adapt to tolerance of communication problems and the ability to avoids the disadvantages of communication at congested area. Selectively: To design an efficient routing protocol, there is a need for designing an algorithm that can select one optimal relay among the multiple available nodes to reduce the data collision probability and minimize the transmission redundancy. Multi-Objective: Current solutions designed for VANET consider only a single objective. The performance optimization of a single objective protocol does not consider varying aspects of the vehicular network. However, designing multi-objective routing protocol is a challenging task because of the need to balance conflicting trade-offs between several routing metrics. Adaptability: To design an efficient routing protocol that should adapt for using different application to fulfill the specific QoS requirements with considering several demands such as better packet delivery ratio with low latency and effective use of available bandwidth.

\section{References}

Abbasi, I. A. Nazir, B., \& Abbasi, A. (2014). A traffic flow-oriented routing protocol for VANETs. Journal Wireless Communication Network, 121(2014). https://doi.org/10.1186/1687-1499-2014-121

Ali, F., Shaikh, F. K., Ansari, A. Q., Mahoto, N. A., \& Felemban, E. (2015). Comparative Analysis of VANET Routing Protocols: On Road Side Unit Placement Strategies. Wireless Personal Communications, 85(2), 393-406. https://doi.org/10.1007/s11277-015-2745-z

Ali, K., Phillips, I., \& Yang, H. (2016, June). Evaluating VANET routing in urban environments. 2016 39th International Conference on Telecommunications and Signal Processing (TSP), Vienna, Austria, pp. 60-63. https://doi.org/10.1109/TSP.2016.7760829

Amina, B., \& El Boukhari, M. (2018). Classification and Comparison of Routing Protocols in VANETs. 2018 The International Conference on Intelligent Systems and Computer Vision. https://doi.org/10.1109/ISACV.2018.8354025

Amina, B., Asmae, B., \& Mohamed, E. (2020). Routing Protocols for VANETs: A Taxonomy, Evaluation and Analysis. Advances in Science, Technology and Engineering Systems Journal, 5(1), 77-85. https://doi.org/10.25046/aj050109

Anil, T., Kumar, T. R., \& Sivakumar, T. (2016). A Survey on Unicast Routing Protocols for VANET. International Journal of Engineering and Computer Science, 5(5), 16555-16565. https://doi.org/10.18535/ijecs/v5i5.37

Asim, R., Saira, G., Sana, A., \& Amir, Q. (2017). Vehicular Ad Hoc Network (VANET): A Survey, Challenges, and Applications. In Laouiti, A., Qayyum, A. \& Mohamad Saad, M. (Eds), Vehicular Ad-Hoc Networks for Smart Cities. Advances in Intelligent Systems and Computing, vol 548. Springer, Singapore. https://doi.org/10.1007/978-981-10-3503-6_4

Bilal, S. M., Khan, A. R., \& Ali, S. (2016). Review and performance analysis of position based routing protocols. Wireless Pers. Area Communication, 1, 559-578. https://doi.org/10.1007/s11277-016-3637-6

Bilal, S. M., Madani, S. A., \& Khan, I. A. (2011). Enhanced junction selection mechanism for routing protocol in VANETs. Arab Journal Information Technology, 8(4), 422-429. 
Debasis, D., \& Rajiv, M. (2018). Improvised dynamic network connectivity model for Vehicular Ad-Hoc Networks (VANETs). Journal of Network and Computer Applications, 122, 107-114. https://doi.org/10.1016/j.jnca.2018.08.014

Fabrizio, G., Giulia, B., Dzmitry, K., \& Gianni, V. (2007). Enhanced GPSR Routing in Multi-Hop Vehicular Communications through Movement Awareness. IEEE Communications Letters, 11(10), 781-783. https://doi.org/10.1109/LCOMM.2007.070685

Forderer, D. (2005). Street-Topology Based Routing. Master's thesis, University of Mannheim.

Giudici, F., \& Pagani, E. (2005). Spatial and Traffic-Aware Routing (STAR) for Vehicular Systems. In Yang, L.T., Rana, O. F., Di Martino, B. \& Dongarra, J. (Eds.), High Performance Computing and Communications. HPCC 2005. Lecture Notes in Computer Science, vol 3726. Springer, Berlin, Heidelberg. https://doi.org/10.1007/11557654_11

Gong, J., Xu, C., \& Holle, J. (2007, June). Directional Greedy Routing in Vehicular Ad hoc Networks. 27th International Conference on Distributed Computing Systems Workshops (ICDCSW'07), Toronto, Ont., 2007, 2-2. https://doi.org/10.1109/ICDCSW.2007.65

Harinder, K., \& Meenakshi. (2017, May). Analysis of VANET geographic routing protocols on real city map. 2nd IEEE International Conference on Recent Trends in Electronics, Information \& Communication Technology (RTEICT), Bangalore, 895-899. https://doi.org/10.1109/RTEICT.2017.8256727

De-Ling Huang, Chang Su, Yu-Song Yan, \& Guang-Xia Xu. (2016, September). A Dynamic Hop Choosing Mechanism for Routing in VANETs. 2016 International Conference on Artificial Intelligence: Techniques and Applications (AITA 2016), 25, 2016, pp. 110-114, Shanghai. https://doi.org/10.12783/dtcse/aita2016/7553

Jerbi, M., Senouci, SM., Meraihi, R., \& Ghamri-Doudane, Y. (2007, June). An Improved Vehicular Ad Hoc Routing Protocol for City Environments. 2007 IEEE International Conference on Communications, Glasgow, UK, 2007, 3972-3979. https://doi.org/10.1109/ICC.2007.654

Joanne, S. (2017). Adaptive Routing Protocols for VANET. A Dissertation, Florida Atlantic University.

Joilson, A. J. Emilio, C., \& Wille, G. (2018). Routing in Vehicular Ad Hoc Networks: Main Characteristics and Tendencies. Journal of Computer Network sand Communications. https://doi.org/10.1155/2018/1302123

Karp, B., \& Kung, H. T. (2000, September). GPSR: Greedy Perimeter Stateless Routing for Wireless Networks. Proceedings of the Annual International Conference on Mobile Computing and Networking, Mobicom, 2000. https://doi.org/10.1145/345910.345953

Kirsch, B., \& Effelsberg, W. (2007). Implementation of a Distance-Vector-Based Recovery-Strategy for Position-Based-Routing. Thesis, Department of Mathematics and Computer Science, University of Mannheim. Retrieved from https://ir.uz.ac.zw/jspui/bitstream/10646/865/1/Sambo_thesis.pdf

Kumar, R., \& Rao, S. V. (2008, December). Directional Greedy Routing Protocol (DGRP) in Mobile Ad-Hoc Networks. 2008 International Conference on Information Technology, Bhubaneswar, 2008. pp. 183-188. https://doi.org/10.1109/ICIT.2008.28

Lee, K. C., Le, M., Harri, J., \& Gerla, M. (2008, September). LOUVRE: Landmark Overlays for Urban Vehicular Routing Environments. 2008 IEEE 68th Vehicular Technology Conference, Calgary, AB, Canada, 2008, pp. 1-5. https://doi.org/10.1109/VETECF.2008.447

Lee, K. C., Haerri, J., Lee, U., \& Gerla, M. (2007, November). Enhanced perimeter routing for geographic forwarding protocols in Urban Vehicular Scenarios. 2007 IEEE Globecom Workshops, 2007, pp. 1-10. https://doi.org/10.1109/GLOCOMW.2007.4437832

Lochert, C. Mauve, M. Füßler, H., \& Hartenstein, H. (2005). Geographic routing in city scenarios. ACM SIGMOBILE Mobile Computing and Communications Review, 9(1), 69-72. https://doi.org/10.1145/1055959.1055970

Lochert, C., Mauve, M., Füßler, H., \& Hartenstein, H. (2003, July). A routing strategy for vehicular ad hoc networks in city environments. IEEE IV2003 Intelligent Vehicles Symposium. Proceedings (Cat. No.03TH8683), Columbus, OH, USA, 2003, pp. 156-161. https://doi.org/10.1109/IVS.2003.1212901

Manvendra, S. (2015). Non-DTN Geographic Unicast Routing Protocol for VANET: State of the art. International Journal of Current Engineering and Technology, 5(5), 3418-3425. 
Naumov, V., \& Gross, T. R. (2007, May). Connectivity-Aware Routing (CAR) in Vehicular Ad-hoc Networks. IEEE INFOCOM 2007, 26th IEEE International Conference on Computer Communications, 1919-1927. https://doi.org/10.1109/INFCOM.2007.223

Naumov, V., Baumann, R., \& Gross, T. (2006, May). An evaluation of inter-vehicle ad hoc net-works based on realistic vehicular traces. Proceedings of the 7th ACM Inter-national Symposium on Mobile Ad Hoc Networking and Computing (MobiHoc), Florence, Italy, 108-119. https://doi.org/10.1145/1132905.1132918

Prasanth, K., Duraiswamy, K., Jayasudha, K., \& Chandrasekar, C. (2010). Improved packet forwarding approach in Vehicular ad hoc networks using RDGR algorithm. International Journal of Next-Generation Networks, 2(1), 64-77. https://doi.org/10.5121/ijngn.2010.2106

Ram, A., \& Mishra, M. K. (2017). Position Based Density Conscious Routing Protocol in Vehicular Ad Hoc Networks. International Journal of Future Generation Communication and Networking, 10(9), 57-74. https://doi.org/10.14257/ijfgen.2017.10.9.06

Ryu, M. W. (2011). Position-based Routing Algorithm for Improving Reliability of Inter-Vehicle Communication. ACM Transactions on Interactive Intelligent Systems, 5(8), 1388-1403. https://doi.org/10.3837/tiis.2011.08.002

Sascha, S., \& Wolfgang, E. (2008). Position-based unicast routing for city scenarios. 2008 International Symposium on a World of Wireless, Mobile and Multimedia Networks, pp. 1-8. https://doi.org/10.1109/WOWMOM.2008.4594851

Seet, BC., Liu, G., Lee, BS., Foh, CH., Wong, KJ., \& Lee, KK. (2004). A-STAR: A Mobile Ad Hoc Routing Strategy for Metropolis Vehicular Communications. In Mitrou, N., Kontovasilis, K., Rouskas, G. N., Iliadis I. \& Merakos, L. (Eds.), Networking 2004. Lecture Notes in Computer Science, vol 3042. Springer, Berlin, Heidelberg. https://doi.org/10.1007/978-3-540-24693-0_81

Sharef, B. T., Alsaqour, R. a., \& Ismail, M. (2014). Vehicular communication ad hoc routing protocols: A survey. Journal of Network and Computer Applications, 40(1), 363-396. https://doi.org/10.1016/j.jnca.2013.09.008

Shelly, S., \& Babu, A. V. (2015). Link reliability-based greedy perimeter stateless routing for vehicular ad hoc networks. International Journal of Vehicular Technology. https://doi.org/10.1155/2015/921414

Singh, P. (2014, February). Comparative Study Between Unicast and Multicast Routing Protocols in Different Data Rates Using Vanet. 2014 International Conference on Issues and Challenges in Intelligent Computing Techniques (ICICT), Ghaziabad, India. 278-284. https://doi.org/10.1109/ICICICT.2014.6781293

Smiri, S., Boushaba, A., Ben A. R., \& Zahi, A. (2018, April). Geographic and topology based routing protocols in vehicular ad-hoc networks: Performance evaluation and QoS analysis. 2018 International Conference on Intelligent Systems and Computer Vision (ISCV), Fez, Morocco, pp. 1-8. https://doi.org/10.1109/ISACV.2018.8354070

Sunder, A. R., Manohara, P., Mounir, B., \& Joseph, M. (2008, January). GPSR-L: Greedy perimeter stateless routing with lifetime for VANETS. 2008 8th International Conference on ITS Telecommunications, Phuket, Thailand, 299-304. https://doi.org/10.1109/ITST.2008.4740275

Tian, J., Han, L., \& Rothermel, K. (2003, October). Spatially aware packet routing for mobile ad hoc inter-vehicle radio networks. Proceedings of the 2003 IEEE International Conference on Intelligent Transportation Systems, Shanghai, China, 1546-1551. https://doi.org/10.1109/ITSC.2003.1252743

Zineb, S. H., \& Imane, Z. (2017). Comparative Study of Routing Protocols Performance for Vehicular Ad-hoc Networks. International Journal of Applied Engineering Research, 12(13), 3867-3878.

\section{Copyrights}

Copyright for this article is retained by the author(s), with first publication rights granted to the journal.

This is an open-access article distributed under the terms and conditions of the Creative Commons Attribution license (http://creativecommons.org/licenses/by/4.0/). 